\title{
THE LITTLEWOOD-PALEY THEORY FOR JACOBI EXPANSIONS
}

BY

WILLIAM C. CONNETT AND ALAN L. SCHWARTZ ${ }^{1}$

\begin{abstract}
The machinery for harmonic analysis utilizing Jacobi polynomial expansions is developed using the explicit form of the convolution kernel discovered by Gasper. Various maximal functions, and the standard Littlewood-Paley functionals are studied and an application is given to multiplier theorems.
\end{abstract}

1. Introduction. The Littlewood-Paley theory involves certain statements about the $L^{p}$ norm of various functionals constructed out of derivatives of Abel means and differences of Cesàro sums. The theory was originally used to study some of the subtler properties of trigonometric series, but later it was extended to the Fourier transform in $R^{n}$ and then to expansions related to various Lie groups, symmetric spaces, and some weighted measure spaces (see for example [19], [18], and [8]).

In this paper the theory is extended to Jacobi series. The major tool for this extension is the convolution kernel studied by Gasper in [9] and [10]. The major part of what follows is the development of the interplay between the convolution kernel and the Poisson kernel or the Cesàro kernel, or the role of the convolution in various maximal theorems. The paper is divided as follows: In $\$ 2$ the convolution structure is introduced and the various operators $g_{\delta}, g_{\eta}^{*}, S, \gamma_{\delta}$, and $\gamma_{\eta}^{*}$ are defined. Theorem 1 describes the range of validity of the standard inequalities. The proof of this theorem uses a number of other results which are established in $\$ \$ 2$ and 3. In particular Theorems 2 and 3 are results about Cesàro summability. Theorems 4, 5, and 6 study the interplay of the convolution structure and the various maximal functions.

In $\S 4$ the $g_{\delta}, g_{\eta}^{*}$, and $S$ functionals are used to prove a multiplier theorem for the Banach space $S(2, \alpha+1)$ acting on Jacobi series (see $\$ 4$ for definitions). This theorem of Hörmander type extends and improves the earlier results in [7] and [8] and is then used as an end point result along with the results of Askey [1] and Gasper and Trebels [12] to obtain in \$4 a general multiplier theorem which describes a set of parameters $(1 / p, 1 / q, \alpha)$ for which $S(q, \alpha)$ is an $L^{p}$ multiplier for Jacobi expansions.

Presented to the Society, January 4, 1978 under the title The Littlewood-Paley theory for Jacobi expansions and January 7, 1978 under the title Maximal functions and the harmonic structure of Jacobi series; received by the editors January 16, 1978.

'Supported by a Summer Research Grant from the University of Missouri, St. Louis. 
2. The Littlewood-Paley theory for Jacobi expansions. In all that follows, assume $\alpha>\beta>-1$. Define $L^{p}$ as the collection of all $f$ for which

$$
\|f\|_{p}=\left[\int_{0}^{\pi / 2}|f(\theta)|^{p} d \mu\right]^{1 / p}<\infty
$$

where $d \mu(\theta)=2^{\alpha+\beta+2} \sin ^{2 \alpha+1} \theta \cos ^{2 \beta+1} \theta d \theta$. The Jacobi polynomials are the polynomials with argument $\cos 2 \theta, P_{n}^{(\alpha, \beta)}(\cos 2 \theta)$ orthogonal with respect to $d \mu$, normalized so that $P_{n}^{(\alpha, \beta)}(1)=\left(\begin{array}{c}n+\alpha \\ \alpha\end{array}\right)$. Define

$$
R_{n}(\theta)=\frac{P_{n}^{(\alpha, \beta)}(\cos 2 \theta)}{P_{n}^{(\alpha, \beta)}(1)}
$$

so that (cf. Szegö [20, 7.32.2])

$$
-1<R_{n}(\theta)<R_{n}(0)=1 \quad\left(\alpha>-\frac{1}{2}\right) .
$$

The Jacobi series of a function $f$ in $L^{1}$ is given by

$$
f(\theta) \sim \sum_{n=0}^{\infty} \hat{f}(n) h_{n} R_{n}(\theta)
$$

where

$$
\hat{f}(n)=\int_{0}^{\pi / 2} f(\theta) R_{n}(\theta) d \mu(\theta)
$$

and

$$
h_{n}=\left[\int_{0}^{\pi / 2} R_{n}^{2}(\theta) d \mu(\theta)\right]^{-1} \simeq C n^{2 \alpha+1} .
$$

The convolution structure is based on the work of Gasper [10]. A necessary and sufficient condition that there exist a unique measure $m_{\theta, \phi}(\psi)$ such that

(i) $R_{n}(\theta) R_{n}(\phi)=\int_{0}^{\pi / 2} R_{n}(\psi) d m_{\theta, \phi}(\psi)$,

(ii) $\int\left|d m_{\theta, \phi}(\psi)\right|<K$. (uniformly in $\left.\theta, \phi\right)$

is that $\alpha+\beta \geq-1$. The measure is nonnegative if and only if $\beta>-\frac{1}{2}$ or $\alpha+\beta>0$. An important property of this measure is

(iii) $\operatorname{supp} m_{\theta, \phi}(\psi) \subset[|\theta-\phi|, \theta+\phi]$.

Using this measure it is possible to define a convolution

$$
f * g(\theta)=\int_{0}^{\pi / 2} \int_{0}^{\pi / 2} f(\phi) g(\psi) d m_{\theta, \phi}(\psi) d \mu(\phi)
$$

and it follows that, if $f, g \in L^{1}$,

$$
(f * g)^{\wedge}(n)=\hat{f}(n) \hat{g}(n),
$$

and if $1 \leqslant r, p, q \leqslant \infty$ with $1 / r=1 / p+1 / q-1$. Then

$$
\|f * g\|_{r} \leqslant K\|f\|_{p}\|g\|_{q} \text {. }
$$

If $\delta_{\phi}$ is the unit mass concentrated at $\phi$, then a generalized translation is 
defined by

$$
f * \delta_{\phi}(\theta)=\int f(\psi) d m_{\theta, \phi}(\psi)
$$

The Littlewood-Paley theory depends on manipulating certain derivatives or differences of "mollified" functions. The $g$-functions are defined in terms of Abel means and their derivatives. The function

$$
P_{r}(\theta)=\sum r^{n} h_{n} R_{n}(\theta)
$$

is called the untranslated Poisson kernel, the function

$$
P_{r}(\theta, \phi)=\sum r^{n} h_{n} R_{n}(\theta) R_{n}(\phi)
$$

is called the translated Poisson kernel. It is clear that $P_{r}(\theta, 0)=P_{r}(\theta)$, and, if $\alpha+\beta>-1, P_{r}(\theta, \phi)=P_{r} * \delta_{\phi}(\theta)$. The Abel mean of $f$ is given by

$$
f(r, \theta)=\int_{0}^{\pi / 2} f(\phi) P_{r}(\theta, \phi) d \mu(\phi)=\sum r^{n} \hat{f}(n) h_{n} R_{n}(\theta) .
$$

Again if $\alpha+\beta>-1, f(r, \theta)=f * P_{r}(\theta)$. The radial derivative is defined by

$$
d_{\delta} f(r, \theta)=\left(r \frac{\partial}{\partial r}\right)^{\delta} f(r, \theta)
$$

if $\delta$ is an integer, and if $\delta=k-\beta, 0<\beta<1$,

$$
d_{\delta} f(r, \theta)=\frac{1}{\Gamma(\beta)} \int_{0}^{1} d_{k} f(r t, \theta)|\log t|^{\beta-1} \frac{d t}{t} .
$$

A direct calculation then shows that

$$
d_{\delta} f(r, \theta)=\sum n^{\delta} r \hat{f}(n) h_{n} R_{n}(\theta)
$$

and $d_{\delta} d_{\eta} f=d_{\delta+\eta} f$.

For all $\alpha, \beta>-1$, define

$$
g_{\delta}(f, \theta)=\left\{\int_{0}^{1}\left|d_{\delta}(f, \theta)\right|^{2}|\log r|^{2 \delta-1} \frac{d r}{r}\right\}^{1 / 2}
$$

and for $\alpha+\beta \geqslant-1$, define

$$
\begin{aligned}
& S(f, \theta)=\left\{\int_{0}^{1}\left|d_{1} f(r, \cdot)\right|^{2} * \chi_{1-r}(\theta)(1-r)^{-2 \alpha-1} d r\right\}^{1 / 2}, \\
& g_{\eta}^{*}(f, \theta)=\left\{\int_{0}^{1}\left|d_{1} f(r, \cdot)\right|^{2} * k_{\eta, r}(\theta)(1-r)^{-2 \alpha-1} d r\right\}^{1 / 2}
\end{aligned}
$$

where $\chi_{1-r}$ is the characteristic function of the interval $[0,1-r]$ and

$$
k_{\eta, r}(\theta)=\left(\frac{1-r}{1-r+\theta}\right)^{\eta(2 \alpha+2)}
$$


The $\gamma$-functions of Bonami and Clerc [4] are defined in terms of the Cesàro sums:

$$
\begin{aligned}
S_{L}^{\delta} f & =\frac{1}{A_{L}^{\delta}} \sum_{l=0}^{L} A_{L-l}^{\delta} h_{l} \hat{f}(l) R_{l}, \\
A_{L}^{\delta} & =\frac{\Gamma(L+\delta+1)}{\Gamma(L+1) \Gamma(\delta+1)} .
\end{aligned}
$$

These Cesàro sums can be represented by integration against a kernel. Define

$$
K_{L}^{\delta}(\theta, \phi)=\frac{1}{A_{L}^{\delta}} \sum_{l=0}^{L} A_{L-l}^{\delta} h_{l} R_{l}(\theta) R_{l}(\phi),
$$

and it follows that

$$
S_{L}^{\delta} f(\theta)=\int f(\phi) K_{L}^{\delta}(\theta, \phi) d \mu(\phi)
$$

If $\alpha+\beta>-1$, define

$$
K_{L}^{\delta}(\theta)=K_{L}^{\delta}(\theta, 0)
$$

then

$$
K_{L}^{\delta}(\theta, \phi)=K_{L}^{\delta} * \delta_{\phi}(\theta), \quad S_{L}^{\delta} f(\theta)=f * K_{L}^{\delta}(\theta) .
$$

The $\gamma$-functions are defined in terms of certain differences of these sums:

$$
\begin{aligned}
& \gamma_{\delta}(f, \theta)=\left\{\sum_{L=0}^{\infty}\left|S_{L}^{\delta+1} f(\theta)-S_{L}^{\delta} f(\theta)\right|^{2} L^{-1}\right\}^{1 / 2}, \\
& \gamma_{\delta}^{*}(f, \theta)=\left\{\sum_{L=0}^{\infty}\left|S_{L}^{\delta+1} f(\theta)-S_{L}^{\delta} f(\theta)\right|^{2} V_{L} L^{-1}\right\}^{1 / 2}
\end{aligned}
$$

where it is assumed that the sequence $V_{L}$ used in defining $\gamma_{\delta}^{*}$ always satisfies: (a) $V_{L}>0$, (b) $\sup _{L>1} L^{-1} \sum_{k=1}^{L} V_{K}=M<\infty$. (N.B. Bonami and Clerc call these functions $g_{\delta}$ and $g_{\delta}^{*}$, respectively. The older notation using $\gamma$ 's is from Zygmund [22, II, p. 222].)

The Littlewood-Paley theory for the Jacobi expansions then consists of the following statements:

THEOREM 1. If $f \in L^{2} \cap L^{p}$ and $\int f d \mu=0$, then
(a) $\left\|g_{1}(f, \cdot)\right\|_{p}<A_{p}\|f\|_{p}, 1<p<\infty, \alpha, \beta>-1$;
(b) $\|f\|_{p}<A_{p}\left\|g_{1}(f, \cdot)\right\|_{p}, 1<p<\infty, \alpha, \beta>-1$;
(c) $\left\|g_{\eta}^{*}(f, \cdot)\right\|_{p}<A_{p}\left\|g_{1}(f, \cdot)\right\|_{p}, 2<p<\infty, \eta>1, \alpha+\beta>-1$;
(d) $g_{\delta}(f, \theta)<A_{\delta, \eta} g_{\eta}(f, \theta), \eta>\delta, \alpha+\beta>-1$;
(e) $S(f, \theta)<A_{\eta} g_{\eta}^{*}(f, \theta), \eta>0, \alpha+\beta>-1$;
(f) $g_{1}(f, \theta)<C \gamma_{\delta}(f, \theta), \delta>0, \alpha+\beta>0$ or $\beta>-\frac{1}{2}$; 
(g) if $\alpha+\beta \geqslant 0$ or $\beta \geqslant-\frac{1}{2}$, and if $\delta>0$ and $p$ satisfy $\left|1 / p-\frac{1}{2}\right|<(2 \delta+$ 1) $/(4 \alpha+1)$, then $\left\|\gamma_{\delta}(f, \cdot)\right\|_{p} \leqslant C\|f\|_{p}$;

(h) under the assumptions as in $(\mathrm{g}),\left\|\gamma_{\delta}^{*}(f, \cdot)\right\|_{p}<\left\|g_{1}(f, \cdot)\right\|_{p}$.

The relations (a) and (b) are demonstrated by defining the Poisson semigroup

$$
\begin{aligned}
T^{t} f(\theta) & =\int_{0}^{\pi / 2} f(\phi) P_{e^{-t}}(\theta, \phi) d \mu(\phi) \\
( & \left.=f * P_{e^{-t}}(\theta) \text { if } \alpha+\beta \geqslant-1\right)
\end{aligned}
$$

and verifying that $T^{t}$ satisfies the four axioms for a symmetric diffusion semigroup given by Stein [18] as:

I. Contraction: $\left\|T^{t} f\right\|_{p} \leqslant\|f\|_{p}, 1<p<\infty$;

II. Symmetry: each $T^{t}$ is selfadjoint on $L^{2}$;

III. Positivity: $T^{t} f \geqslant 0$ if $f \geqslant 0$;

IV. Conservation: $T^{t} 1=1$.

Then (a) and (b) follow from [18, pp. 111, 120].

Property III follows from the fact that $P_{r}(\theta, \phi)$ is always positive, $\alpha+\beta>$ -2 , even if the convolution measure is not positive. This can be seen from the formula

$$
\begin{aligned}
& P_{r}(\theta, \phi)=\frac{\Gamma(\alpha+\beta+2)(1-r)}{2^{\alpha+\beta+2} \Gamma(\alpha+1) \Gamma(\beta+1)(1+r)^{\alpha+\beta+2}} \\
& \cdot F_{4}\left(\frac{1}{2}(\alpha+\beta+2), \frac{1}{2}(\alpha+\beta+3) ; \alpha+1, \beta+1 ; \frac{a^{2}}{k^{2}}, \frac{b^{2}}{k^{2}}\right)
\end{aligned}
$$

where $a=\sin \theta \sin \phi, b=\cos \theta \cos \phi$, and $k=\frac{1}{2}\left(r^{-1 / 2}+r^{1 / 2}\right)$ (Bailey [2, p. 102]). The definition of the Poisson kernel implies $\int P_{r}(\theta, \phi) d \mu(\theta)=$ $r^{0} h_{0}\left\|R_{0}^{2}\right\| R_{0}(\phi)=1$ which is IV.

Property I follows from the inequality

$$
\left\|P_{r} f(\cdot)\right\|_{p} \leqslant\|f\|_{p}\left\|P_{r}(\theta, \cdot)\right\|_{1} \leqslant\|f\|_{p},
$$

by an adaption of the argument for Young's inequality [16, p. 7], and II follows by a simple change in the order of integration.

The relation (c) is established in $\$ 3$ and it holds in the region where the convolution is bounded on $L^{1}$, in particular for $\alpha+\beta>-1$.

Relations (d) and (e) were proved in [8, p. 66] for the ultraspherical setting and that proof carries over directly to all expansions $\alpha+\beta>-1$.

Relations (f), (g), and (h) are given in [4] for $\alpha=\beta$ and for a few geometric cases. Gasper's work on the Jacobi convolution can be used to extend these inequalities to the $(\alpha, \beta)$ region for which the convolution kernel is positive. Bonami and Clerc [4] have shown that (g) and (h) follow from Zygmund's 
inequality (see Theorem 3 infra), thus it suffices to establish that inequality.

Szegô showed [20, p. 258] that for all $\alpha, \beta>-1, \delta>\alpha+\frac{1}{2}$,

$$
\left\|K_{L}^{\delta}\right\|_{1} \leqslant B \quad \text { (uniformly in } L \text { ). }
$$

Therefore, for $\alpha+\beta>-1$, relation (2.1) yields

$$
\left\|S_{L}^{\delta} f\right\|_{p}=\left\|f * K_{L}^{\delta}\right\|_{p} \leqslant K\left\|K_{L}^{\delta}\right\|_{1}\|f\|_{p}<K B\|f\|_{p}
$$

The constant $K B$ is independent of $L$, and when such an inequality holds $L^{p}$ is said to be $(C, \delta)$ summable. This is the full range result. When $\delta=0$, Pollard [17] showed that $L^{p}$ is $(C, 0)$ summable if

$$
\left|\frac{1}{p}-\frac{1}{2}\right|<\frac{1}{4(\alpha+1)}<\frac{1}{4(\beta+1)} .
$$

Therefore by the interpolation of analytic families of operators [19, p. 239], this proves

THEOREM 2. If $\alpha+\beta \geqslant-1$, then $L^{p}$ is $(C, \delta)$ summable for all $p$ such that

$$
\left|\frac{1}{p}-\frac{1}{2}\right|<\frac{2 \delta+1}{4(\alpha+1)} \quad(\delta>0)
$$

That is,

$$
\left\|S_{L}^{\delta} f\right\|_{p}<C\|f\|_{p}
$$

where the constant depends on $\alpha, p, \delta$ but not $L$.

The proof of the above theorem merely depends on the existence of a bounded convolution. In order to prove Zygmund's inequality, it is necessary to restrict $\alpha$ and $\beta$ so that the convolution measure becomes positive. In particular,

THEOREM 3. For all sequences of functions $\left\{f_{k}\right\}$ and all sequences of integers $\left\{n_{k}\right\}$,

$$
\left\|\left(\sum_{0}^{\infty}\left|S_{n_{k}}^{\delta} f_{k}\right|^{2}\right)^{1 / 2}\right\|_{p}<A_{p}\left\|\left(\sum_{0}^{\infty}\left|f_{k}\right|^{2}\right)^{1 / 2}\right\|_{p}
$$

if $\alpha+\beta>0$ or $\beta \geqslant-\frac{1}{2}$, and $\delta$ and $p$ satisfy

$$
\left|\frac{1}{p}-\frac{1}{2}\right|<\frac{2 \delta+1}{4(\alpha+1)} \quad(\delta>0) .
$$

The proof is adapted from Bonami and Clerc [4]. A full range result is established for $\delta>\alpha+\frac{1}{2}$ by showing that the maximal operator $\sup _{L} S_{L}^{\delta} f(x)=S_{*}^{\delta} f(x)$ is a bounded $L^{p}$ operator $1<p<\infty$ (this is proved in $\S 3$, Theorem 7). The rest of the proof is the same as in [4]. 
If $0>\delta>\alpha+\frac{1}{2}$, then $L^{2}$ is not $(C, \delta)$ summable even though (2.3) still holds. To see this observe that $S_{L}^{\delta}$ is given by a sequence of multipliers $m_{L}^{l}=A_{L-l}^{\delta} / A_{L}^{\delta}$, and $m_{L}^{L} \simeq L^{-\delta}$ which is unbounded.

If $-\frac{1}{2}<\alpha, \alpha+\beta<-1$, then this objection does not apply, and it is conceivable that, as in the Poisson case, $\iint\left|K_{L}^{\delta}(\theta, \phi)\right| d \mu(\theta) d \mu(\phi) \leqslant K$ uniformly in $L$, even though the convolution measure is unbounded (for this see Gasper [10, pp. 263, 278 (6.13)]). In any event, the range of validity for (g) and $(\mathrm{h})$ is not as extensive as the range for (a) and (b).

3. Maximal functions and the harmonic structure of Jacobi expansions. If $f(\theta)>0$ is periodic on $[-\pi, \pi]$, then the maximal function of HardyLittlewood is defined by

$$
f^{\#}(\phi)=\sup _{\varepsilon>0} \frac{1}{2 \varepsilon} \int_{\phi-\varepsilon}^{\phi+\varepsilon} f(\theta) d \theta .
$$

Define the function $h_{\varepsilon}(\theta)=(2 \varepsilon)^{-1} \chi_{[-\varepsilon, \varepsilon]}(\theta)$, where $\chi_{[-\varepsilon, \varepsilon]}$ is the characteristic function of $[-\varepsilon, \varepsilon]$, and extend it periodically, then (3.1) can be rewritten as a convolution:

$$
f^{\#}(\phi)=\sup _{\varepsilon>0} h_{\varepsilon} * f(\phi)=\sup _{\varepsilon>0} \int h_{\varepsilon}(\phi-\theta) f(\theta) d \theta
$$

If $f(r, \theta)$ is the Abel mean of $f(\theta)$, then a second maximal function can be defined by

$$
P_{*} f(\phi)=\sup _{0<r<1} f(r, \phi) .
$$

It is possible to show that there exist positive constants $A$ and $B$ such that

$$
A f^{\#}(\phi) \leqslant P_{*} f(\phi)<B f^{\#}(\phi)
$$

(see for example [19, p. 59]).

The generalization of these ideas to the Jacobi setting leads to three different maximal functions, two of which are equivalent when the convolution is nonnegative. The first maximal function is

$$
M f(\phi)=\sup _{\varepsilon>0} \frac{1}{\mu[\phi-\varepsilon, \phi+\varepsilon]} \int_{\phi-\varepsilon}^{\phi+\varepsilon}|f(\theta)| d \mu(\theta) ;
$$

the second is defined where there is a bounded convolution, i.e. when $\alpha+\beta>-1$ :

$$
\mathscr{T} f(\phi)=\sup _{\varepsilon>0} \iint h_{\varepsilon}(\psi)|f(\theta)|\left|d m_{\phi, \theta}(\psi)\right| d \mu(\theta)
$$

where now $h_{\varepsilon}(\theta)=\chi_{[0, \varepsilon]}(\theta) / \mu[0, \varepsilon]$. If $\alpha+\beta \geqslant 0$ or $\beta>-\frac{1}{2}, d m_{\theta, \phi}(\psi)$ is positive and this integral can be written $h_{\varepsilon} *|f|(\phi)$, and in any case $h_{\varepsilon} *|f|(\phi)$ is bounded by the double integral. The third maximal function is analogous to (3.3): 


$$
P_{*} f(\phi)=\sup _{0<r<1}|f(r, \phi)|=\sup _{0<r<1}\left|\int_{0}^{\pi / 2} f(\theta) P_{r}(\theta, \phi) d \mu(\theta)\right| .
$$

$\mathfrak{N} f$ and $M f$ are not obviously related since $\mu[\phi-\varepsilon, \phi+\varepsilon] / \mu[0, \varepsilon]$ is not a bounded ratio; however, the $L^{p}$ inequalities for $M f$ will follow from

THEOREM 4. If $\alpha+\beta \geqslant-1$, then there exists $K>0$ such that

$$
\mathfrak{N} f(\phi)<K M f(\phi) \text {. }
$$

Proof. The special case $\alpha=\beta$ is equivalent to the lemma in $[6$, p. 63], thus it will be assumed below that $\alpha>\beta$. The proof in the Jacobi setting requires even more knowledge of special functions to carry through the details, in particular a formula due to Macdonald [15] for the representation of a triple integral of Bessel functions as an associated Legendre function (this is also in Watson [21, p. 412]; see Gasper [10, p. 268] and Koornwinder [14, p. 135] for more modern presentations of this formula and its application to Jacobi expansions).

Let $f \geq 0$; define

$$
\mathfrak{K}_{\varepsilon} f(\phi)=\frac{1}{\mu[0, \varepsilon]} \int_{\phi-\varepsilon}^{\phi+\varepsilon} f(\theta) \int_{|\phi-\theta|}^{\varepsilon}\left|d m_{\theta, \phi}(\psi)\right| d \mu(\theta)
$$

and

$$
M_{\varepsilon} f(\phi)=\frac{1}{\mu[\phi-\varepsilon, \phi+\varepsilon]} \int_{\phi-\varepsilon}^{\phi+\varepsilon} f(\theta) d \mu(\theta) .
$$

Thus it suffices to show that $\mathfrak{K}_{e} f(\phi)<K M_{\varepsilon} f(\phi)$, with the constant independent of $\varepsilon$. This will follow immediately from

$$
J=\mu(\phi-\varepsilon, \phi+\varepsilon) \int_{|\phi-\theta|}^{\varepsilon}\left|d m_{\theta, \phi}(\psi)\right|<K \mu[0, \varepsilon] \simeq C \varepsilon^{2 \alpha+2}
$$

There are a number of cases to consider:

(i) $\alpha+\beta>-1,-\frac{1}{2}<\alpha<\frac{1}{2}$,

(ii) $\alpha+\beta>-1, \alpha=\frac{1}{2}$,

(iii) $\alpha+\beta>-1, \alpha>\frac{1}{2}$,

(iv) $\alpha+\beta=-1$ (the singular case).

In each case, the formula for $d m_{\theta, \phi}(\psi)$ is given explicitly by Gasper [10, formulas (1.12), (3.3), (3.4), (6.4)] and is different. In each case the evaluation of the integral in (3.7) involves considering typical values of the variable: $\phi<3 \varepsilon, 3 \varepsilon<\phi<\pi / 2-3 \varepsilon, \phi>\pi / 2-3 \varepsilon$, and for each of these ranges of the variables, the argument is different. Since there are many cases, and so many of the computations are similar, only the computations needed to verify case (ii) will be given in detail here-it was chosen because of a logarithmic singularity which is not present in the other cases. 
Let $\alpha=\frac{1}{2}, a=\sin \phi \sin \theta, b=\cos \phi \cos \theta, c=\cos \psi, B=\left(b^{2}+c^{2}-\right.$ $\left.a^{2}\right) / 2 b c$, then Gasper shows that $d m_{\theta, \phi}(\psi)=G(\theta, \phi, \psi) d \mu(\psi)$ with

$$
\begin{aligned}
G(\theta, \phi, \psi)= & \frac{\Gamma\left(\frac{3}{2}\right) a^{-1}\left(1-c^{2}\right)^{-1 / 2}(b c)^{-\beta-1 / 2}}{\Gamma\left(\frac{1}{2}\right) 2^{\beta+5 / 2}} \\
& \cdot F\left[\frac{1}{2}+\beta, \frac{1}{2}-\beta ; 1 ; \frac{1}{2}(1-B)\right]
\end{aligned}
$$

if $|a-b|<c<a+b$, and

$$
\begin{aligned}
G(\theta, \phi, \psi)= & \frac{\Gamma\left(\frac{3}{2}\right) a^{-1}\left(1-c^{2}\right)^{-1 / 2}\left(a^{2}-b^{2}-c^{2}\right)^{-\beta-1 / 2}}{\Gamma\left(\frac{1}{2}-\beta\right) \Gamma(\beta+1) 2^{\beta+3 / 2}} \\
& \cdot F\left[\frac{1}{2}\left(\beta+\frac{1}{2}\right), \frac{1}{2}\left(\beta+\frac{3}{2}\right) ; \beta+1 ; B^{-2}\right]
\end{aligned}
$$

if $c<a-b$, and $G(\theta, \phi, \psi)=0$ if $c<b-a$ or $c>a+b$.

In the other three cases a hypergeometric function $F[\lambda, \gamma ; \nu ; s]$ appears, but because of a theorem of Gauss, $F$ is uniformly bounded for $|s|<1$ provided $\nu-\lambda-\gamma>0$. In the case being considered $\nu-\lambda-\gamma=0$, and by a theorem of Appell (see, for example, Bartle [3, p. 419]), $\lim _{s \rightarrow 1^{-}}(F[\lambda, \gamma ; \nu ; s] / \log (1 /(1-s)))$ exists and is finite.

The support of $G$ requires that $b-a<c<a+b$ which by a change of variables becomes $|\theta-\phi|<\psi<\theta+\phi$. The proof of case (ii) proceeds by considering four cases.

Case (1), $\phi<3 \varepsilon$. This is immediate since

$$
J<\mu[0,4 \varepsilon] \int_{0}^{\pi / 2}\left|d m_{\theta, \phi}(\psi)\right|<C \varepsilon^{2 \alpha+2} .
$$

In all of the cases that follow, $C$ will indicate a constant that does not depend on $\varepsilon$ or $\phi$, but may change from place to place.

The change of variables $c=\cos \psi$ yields

$$
J=\mu[\phi-\varepsilon, \phi+\varepsilon] \int_{\cos \varepsilon}^{a+b}|G(\theta, \phi, \psi)|\left(1-c^{2}\right)^{1 / 2} c^{2 \beta+1} d c .
$$

In all of the remaining cases, $3 \varepsilon<\phi<\pi / 2$, so the following inequalities hold:

$$
\begin{gathered}
a+b-\cos \varepsilon=\cos (\theta-\phi)-\cos \varepsilon<\varepsilon^{2}, \\
a+b>c>\cos \varepsilon>\sqrt{3} / 2 \\
a^{2}-b^{2}-c^{2}<(a+b+c)(a+b-c)<2(a+b-c) \\
>(a-b-c)(a-b+c)>\sqrt{3} / 2(a-b-c) \quad(\text { if } a>b) .
\end{gathered}
$$


Case (2), $3 \varepsilon<\phi<\pi / 2$ and $a<b$. For this case the following inequalities hold:

$$
b>\sqrt{3} / 4, \quad \mu[\phi-\varepsilon, \phi+\varepsilon] \sim \varepsilon a, \quad 1+B \sim 1
$$

(the symbol $x \sim y$ means $x / y$ and $y / x$ are bounded). Now substituting (3.8) in (3.10), assuming $b-a<\cos \varepsilon$ and using Appell's theorem, obtain

$$
\begin{aligned}
J & <C \varepsilon a \int_{\cos \varepsilon}^{a+b}\left\{a^{-1}\left(1-c^{2}\right)^{-1 / 2}(b c)^{-\beta-1 / 2}\right. \\
& \left.\cdot \log \left(\frac{1}{1-\frac{1}{2}(1-B)}\right)\right\}\left(1-c^{2}\right)^{1 / 2} c^{2 \beta+1} d c \\
& <C \varepsilon \int_{\cos \varepsilon}^{a+b} \log \left(2(1+B)^{-1}\right) d c \\
& <C \varepsilon(a+b-\cos \varepsilon)<C \varepsilon^{3} .
\end{aligned}
$$

If $\cos \varepsilon<b-a$, a similar estimate works.

Case (3), $a>b$ and $3 \varepsilon<\phi<\pi / 2-3 \varepsilon$. The following relations now hold:

$$
\begin{gathered}
a>\sqrt{3} / 4, \quad a-b<\cos \varepsilon<c, \quad \mu[\phi-\varepsilon, \phi+\varepsilon] \sim \varepsilon b^{\beta+1 / 2}, \\
1+B \sim(c-(a-b)) / 2 b .
\end{gathered}
$$

It follows again by substitution of (3.8) in (3.10) that

$$
J<C \varepsilon b^{\beta+1 / 2} \int_{\cos \varepsilon}^{a+b} b^{-\beta-1 / 2} \log \left(\frac{2 b}{c-(a-b)}\right) d c
$$

and, since $\int \log x=x(\log x-1)$,

$$
\begin{aligned}
J & <C \varepsilon\{2 b-[\cos \varepsilon-(a-b)]\}\left[\log \frac{2 b}{\cos \varepsilon-(a-b)}+1\right] \\
= & C \varepsilon\{[a+b-\cos \varepsilon]+[\cos \varepsilon-(a-b)] \\
& \cdot[\log 2 b-\log \{\cos \varepsilon-(a-b)\}]\} \\
& <C \varepsilon[a+b-\cos \varepsilon]<C \varepsilon^{3}
\end{aligned}
$$

by the mean value theorem.

Case (4), $a>b$ and $\pi / 2-3 \varepsilon<\phi<\pi / 2$. The following relations hold:

$$
\begin{gathered}
a>\sqrt{3} / 4, \quad b<C \varepsilon^{2}, \quad \mu[\phi-\varepsilon, \phi+\varepsilon] \sim \varepsilon^{2 \beta+2}, \\
a-b-c<\varepsilon^{2} \quad \text { if } \cos \varepsilon<c<a-b, \\
1+B \sim(c-(a-b)) / 2 b,
\end{gathered}
$$


and if $c<a-b, B<-1$ so

$$
1-B^{-2} \sim 1+B^{-1} \sim(a-b-c) /\left(a^{2}-b^{2}-c^{2}\right) .
$$

Now if $\cos \varepsilon<a-b$, then

$$
J \leqslant C \varepsilon^{2 \beta+2} \int_{\cos \varepsilon}^{a+b}=C \varepsilon^{2 \beta+2}\left[\int_{\cos \varepsilon}^{a-b}+\int_{a-b}^{a+b}\right]=\mathrm{I}+\mathrm{II} .
$$

In I, $c<a-b$ so substitution of (3.9) in (3.10) yields

$$
\begin{aligned}
I & \leqslant C \varepsilon^{2 \beta+2} \int_{\cos \varepsilon}^{a-b}\left(a^{2}-b^{2}-c^{2}\right)^{-\beta-1 / 2} \log \left(\frac{1}{1-B^{-2}}\right) d c \\
& \leqslant C \varepsilon^{2 \beta+2} \int_{\cos \varepsilon}^{a-b}\left(a^{2}-b^{2}-c^{2}\right)^{-\beta-1 / 2} \log \left(\frac{1}{1+B^{-1}}\right) d c .
\end{aligned}
$$

Since $B<-1, \log \left(1+B^{-1}\right)^{-1}=-\log \left(1+B^{-1}\right)$ can be expanded in powers of $B^{-1}$ to obtain

$$
\mathrm{I} \leqslant C \varepsilon^{2 \beta+2} \int_{\cos \varepsilon}^{a-b} \sum_{k=1}^{\infty} k^{-1}(2 b c)^{k}\left(a^{2}-b^{2}-c^{2}\right)^{-k-\beta-1 / 2} 2 c d c .
$$

Since $c<a-b$ in the range of integration, Fubini's theorem yields

$$
\left.\mathrm{I}<C \varepsilon^{2 \beta+2}\left(a^{2}-b^{2}-\cos ^{2} \varepsilon\right)^{1 / 2-\beta} \sum_{k=1}^{\infty} k^{-2}\left(\frac{2 b(a-b)}{a^{2}-b^{2}-c^{2}}\right)^{k}\right]_{\cos \varepsilon}^{a-b},
$$

since $\frac{1}{2}-\beta>0$. But $c \leqslant a-b$ implies that $2 b(a-b) /\left(a^{2}-b^{2}-c^{2}\right)<1$ so that the series has an absolute bound independent of $\varepsilon$. Now it is immediate that

$$
\mathrm{I} \leqslant C \varepsilon^{2 \beta+2}\left(\varepsilon^{2}\right)^{1 / 2-\beta}=C \varepsilon^{3} .
$$

Finally, evaluate II by substituting (3.8) in (3.10):

$$
\begin{aligned}
\text { II } & \leqslant C \varepsilon^{2 \beta+2} \int_{a-b}^{a+b} b^{-\beta-1 / 2} \log \frac{1}{1-\frac{1}{2}(1-B)} d c \\
& \leqslant C \varepsilon^{2 \beta+2} b^{-\beta-1 / 2} \int_{a-b}^{a+b} \log \left(\frac{2 b}{c-(a-b)}\right) d c \\
& \leqslant C \varepsilon^{2 \beta+2} b^{1 / 2-\beta}<C \varepsilon^{3} .
\end{aligned}
$$

If $\cos \varepsilon>a-b$, the same methods work with the simplification that only II must be evaluated. This concludes the proof of the theorem.

It remains now to relate the maximal functions $P_{*} f$ and $S_{*}^{\delta}$ to $\Re f$. Since the arguments in these cases are somewhat similar, the development will be simplified by proving the following general theorem, which is an easy adaption of theorems given by Hardy and Littlewood in [13]. 
Let $w_{p}(\theta)$ be an integrable function that depends on a parameter $p$ and define $h(p, \theta)=f * w_{p}(\theta)$.

THEOREM 5. Assume either

(a) there is $a B>0$, independent of $p$ such that

$$
\left|\int_{0}^{\pi / 2} w_{p}(\theta) d \mu(\theta)\right| \leqslant B
$$

and

$$
\int_{0}^{\pi / 2}\left|\mu[0, \theta] \frac{d}{d \theta} w_{p}(\theta)\right| d \theta<B
$$

or

(b) there are functions $W_{p}(\theta)>\left|w_{p}(\theta)\right|$ and $C$ independent of $p$ such that $W_{p}(\pi / 2)<C$ and

$$
\int_{0}^{\pi / 2}\left|\mu[0, \theta] \frac{d}{d \theta} W_{p}(\theta)\right| d \theta<C
$$

then there is $K$ independent of $p$ such that

$$
|h(\theta, p)|<K \Re f(\theta) \text {. }
$$

The proof is based on integration by parts. Let $f_{1}(\psi)=\int_{\delta} f * \delta_{\theta}(\phi) d \mu(\phi)$ so that

$$
\begin{aligned}
h(p, \theta) & =\int_{0}^{\pi / 2} f * \delta_{\theta}(\psi) w_{p}(\psi) d \mu(\psi) \\
& =\left.f_{1}(\psi) w_{p}(\psi)\right|_{0} ^{\pi / 2}-\int_{0}^{\pi / 2} \frac{f_{1}(\psi)}{\mu[0, \psi]} \mu[0, \psi] \frac{d}{d \psi} w_{p}(\psi) d \psi,
\end{aligned}
$$

and proceed as in [13, Theorems 15 and 19].

Theorem 6. Suppose $f$ is nonnegative; then there exist positive constants $A$ and $B$ such that

$$
\begin{aligned}
A \mathscr{N} f(\phi) & <P_{*} f(\phi) \quad \text { if } \alpha+\beta>0 \text { or } \beta>-\frac{1}{2}, \\
P_{*} f(\phi) & \leqslant B \mathfrak{N} f(\phi) \quad \text { if } \alpha+\beta>-1 .
\end{aligned}
$$

Proof. Observe that Stirling's formula implies

$$
\frac{(a)_{n}(b)_{n}}{n !(c)_{n}} / A_{n}^{a+b-c-1} \rightarrow \frac{\Gamma(a+b-c) \Gamma(c)}{\Gamma(a) \Gamma(b)},
$$

so that Appell's theorem yields

$$
(1-s)^{a+b-c} F(a, b ; c ; s) \rightarrow \frac{\Gamma(a+b-c) \Gamma(c)}{\Gamma(a) \Gamma(b)} \quad(a+b-c>0) .
$$


Since the formula of Bailey ((2.2) above) reduces to a hypergeometric series when $\theta=0$,

$$
P_{r}(\phi)=P_{r}(\phi, 0) \sim C \frac{(1-r)}{(1+r)^{\alpha+\beta+2}}\left(1-\frac{\cos ^{2} \phi}{k^{2}}\right)^{-\alpha-3 / 2} .
$$

Now $1-\cos ^{2} \phi / k^{2} \sim\left[(1-r)^{2}+\sin ^{2} \phi\right]$ so finally there must exist positive constants $C_{1}$ and $C_{2}$ such that

$$
C_{1} P_{r}(\phi) \leqslant(1-r)\left[(1-r)^{2}+\sin ^{2} \phi\right]^{-\alpha-3 / 2} \leqslant C_{2} P_{r}(\phi) .
$$

In particular, if $\phi \leqslant(1-r)$,

$$
C_{2} P_{r}(\phi) \geqslant 2^{-\alpha-3 / 2}(1-r)^{-2 \alpha-2} ;
$$

hence, for some positive constant $A, P_{r}(\phi) \geqslant A h_{1-r}(\phi)$. The restrictions on $\alpha$ and $\beta$ are chosen to guarantee a positive convolution so that

$$
f(r, \phi)=f * P_{r}(\phi) \geqslant A f * h_{1-r}(\phi)=A \Re_{1-r} f(\phi) .
$$

To obtain the reverse inequality let $W_{r}(\theta)$ be the middle expression in (3.11) and use Theorem 5.

The following result generalizes a theorem in Bonami and Clerc [4].

TheOREM 7. Suppose $\alpha+\beta \geqslant-1$ and $\alpha+\frac{1}{2}<\delta<\left[\alpha+\frac{3}{2}\right]$ (the greatest integer in $\left.\alpha+\frac{3}{2}\right)$. Then

$$
S_{*}^{\delta} f(\phi) \leqslant A\left[\mathfrak{T} f(\phi)+\iint|f(\theta)|\left|\frac{1}{2} \pi-\psi\right|^{-\beta-1 / 2} d\left|m_{\theta, \phi}(\psi)\right| d \mu(\theta)\right] .
$$

Proof. The following inequalities hold:

$$
\begin{aligned}
& \left|K_{L}^{\delta}(\theta)\right| \leqslant C L^{2 \alpha+2}, \quad 0<\theta<\frac{1}{4} \pi, \\
& \left|K_{L}^{\delta}(\theta)\right| \leqslant C L^{\alpha+1 / 2-\delta}|\theta|^{-\alpha-\delta-3 / 2}, \quad 2 L^{-1} \leqslant \theta<\frac{1}{4} \pi, \\
& \left|K_{L}^{\delta}(\theta)\right| \leqslant C\left|\frac{1}{2} \pi-\theta\right|^{-\beta-1 / 2}, \quad \frac{1}{4} \pi \leqslant \theta \leqslant \frac{1}{2} \pi .
\end{aligned}
$$

The first two relations are found in [4, Theorem 2.1] where the notation $s_{L}^{\delta}(\cos 2 \theta)=K_{L}^{\delta}(\theta)$ is used. The remaining inequality is obtained by the techniques used in the cited work. Now let $w_{L}(\theta)=K_{L}^{\delta}(\theta) x_{[0, \pi / 4]}(\theta)$. The first two relations above then imply that $W_{L}(\theta)=C L^{\alpha+1} / 2-\delta\left(L^{-1}+\right.$ $\theta)^{-(\alpha+\delta+3 / 2)}$ is a majorant for $w_{L}(\theta)$, so the result follows by Theorem 5 .

It is now possible to state the main result of this section.

THEOREM 8. Let $1<p \leqslant \infty$.

(a) For $\alpha, \beta>-1, M$ is of strong type $p-p$ and weak type $1-1$.

(b) For $\alpha+\beta \geqslant-1$, श is of strong type $p-p$ and weak type $1-1$.

(c) For $\alpha+\beta \geqslant-1, P_{*}$ is of strong type $p-p$ and weak type $1-1$.

(d) For $\alpha+\beta>-1$ and $\alpha+\frac{1}{2}<\delta, S_{*}^{\delta}$ is of strong type $p-p$ and weak type $1-1$. 
Proof. (a) Since the interval $[0, \pi / 2]$ with the measure $d \mu$ is of "homogeneous type" the argument in [5, p. 71] yields the result.

(b) By Theorem 4 and the above case, $\Re$ has the desired properties.

(c) In the region $\alpha+\beta \geqslant-1$, Theorem 6 and the above implies the result since for $f$ of arbitrary sign $P_{*} f \leqslant P_{*}|f|<\Re_{f} f$ because $P_{r}(\theta, \phi)>0$.

(d) The argument is similar to (c).

REMARK. An alternate proof that $\mathfrak{T}$ is of strong type $p-p$ can easily be constructed from Theorem 6 and the observation that the semigroup $T^{t} f(\phi)$ $=f\left(e^{-t}, \phi\right)$ satisfies properties I and II of the axioms for a symmetric diffusion semigroup, and by Stein $\left[18\right.$, p. 73] $P_{*}$ must be of strong type $p-p$.

Finally, it is now possible to complete the proof of Theorem 1(c). The argument is based on the same ideas as in [8, p. 62], so that it will suffice to establish that

$$
\|Q F\|_{q}<A\|F\|_{q} \quad(1<q<\infty)
$$

where

$$
Q F(\phi)=\sup _{0<r<1} \int(1-r)^{-2 \alpha-2} k_{\eta, r}(\psi) F * \delta_{\phi}(\psi) d \mu(\psi) .
$$

It suffices to show $Q F(\phi) \leqslant C \Re F(\phi)$ whenever the right-hand side is finite. Let $w_{r}(\theta)=(1-r)^{-2 \alpha-2} k_{\eta, r}(\theta)$ and apply Theorem 5 and Theorem 8(b).

4. Multipliers for Jacobi series. An immediate application of the above ideas is the following multiplier theorem.

THEOREM 9. The Banach space $S(2, \eta)$ is a space of $L^{p}$ multipliers for $1<p<\infty$, if $\eta \geqslant \alpha+1, \alpha>0, \beta>-1$.

The spaces $S(q, \eta)$ are not defined here (see [8, p. 32] for complete definitions and a discussion of the properties of the $S[q, \eta]$ spaces). Suffice it to say that when $k$ is a positive integer, $1 \leqslant q<\infty, m \in S[q, k]$, if and only if

(a) $\sup _{n}|m(n)|=C_{0}<\infty$,

(b) $\sup _{M}\left\{M^{-1} \Sigma_{M}^{2 M}\left|n^{k} \Delta^{k} m(n)\right|^{q}\right\}^{1 / q}=C_{1}<\infty$

and the norm of $m$ in $S[q, k]$ is equivalent to $C_{0}+C_{1}\left(\Delta^{k} m(n)\right.$ is the $k$ th difference of the sequence $\{m(n)\})$. Thus the $S(q, \eta)$ spaces contain as special cases the classical multiplier sequences.

What is needed is the following

LEMMA. If $\eta \geqslant 1, m \in S(2, \eta), m(j)=0$ for $j=0,1, \ldots,[\eta]+1$. Define $M(r, \theta)=\sum_{n=0}^{\infty} m(n) r^{n} h_{n} R_{n}(\theta)$, then

(a) $\left|d_{\eta} M(r, \theta)\right| \leqslant A(1-r)^{-\eta-2 \alpha-2}\|m\|_{\infty}$,

(b) $\left\|(1-\cos \theta)^{\eta / 2} d_{\eta} M(r, \theta)\right\|_{2} \leqslant \operatorname{Ar}(1-r)^{-\alpha-1}\|m\|_{S(2, \eta)}$.

The proof of (a) is an easy consequence of the estimate $h_{n} \sim n^{2 \alpha+1}$ and the 
fact that $m(n)$ is bounded. The proof of (b) is an easy adaptation of the argument in [8]. The only change is that the following lemma must be used in place of $[8$, p. 69 , equation (2)].

LEMMA. Suppose $\eta$ is an integer and $f \sim \sum c_{n} h_{n} R_{n}$; then

$$
\int(1-\cos \theta)^{\eta}[f(\theta)]^{2} d \mu(\theta) \leqslant K \sum_{n=0}^{\infty} \sum_{j=1}^{\eta}\left[(n+1)^{j-\eta} \Delta^{j} c_{n}\right]^{2} h_{n} .
$$

The lemma is similar to [6, Lemma 4.3] but it makes use of the difference computations in $[7, \S 5]$.

The proof of the theorem now follows the well-understood path given in $[8$, p. 73] so it is omitted.

It is now possible to interpolate between the above theorem and the following results:

A. If $m \in S\left(1, \alpha+\frac{3}{2}\right)$, then $m$ defines an $L^{p}$ multiplier for $1<p<\infty$, $\alpha \geqslant \beta \geqslant-\frac{1}{2}$ (Gasper and Trebels [11], [12]).

B. If $m \in S(q, 1 / q+\varepsilon)$, then $m$ is a $L^{2}$ multiplier for $1<q<\infty$.

C. If $m \in S(1,1)$, then $m$ is an $L^{p}$ multiplier for all $p$ such that $\left|1 / p-\frac{1}{2}\right|$ $<1 / 2(2 \alpha+2), \alpha \geqslant \beta \geqslant-\frac{1}{2}$ (Askey [1]).

If $\mathcal{E}_{S}^{\alpha, \beta}$ is defined as the set of parameters $(1 / p, 1 / q, \gamma)$ for which the operator $\mathcal{T}: L^{p} \oplus S(q, \gamma) \rightarrow L^{p}$ defined by $\mathcal{T}(m, f) \sim \sum m(n) \hat{f}(n) h_{n} R_{n}$ is continuous, then $\mathcal{E}_{S}^{\alpha, \beta}$ has a convex interior and contains the above mentioned results as "edge" results. The proof is an immediate application of the ideas of weak-stability and the fact that the $S(q, \gamma)$ spaces are Sobolev modules (see $[8, \S 10]$ for details).

\section{BIBLIOGRAPHY}

1. R. Askey, A transplantation theorem for Jacobi series, Illinois J. Math. 13 (1969), 583-590.

2. W. Bailey, Generalized hypergeometric series, Cambridge Tracts in Mathematics and Mathematical Physics, New York, 1964.

3. R. Bartle, The elements of real analysis, Wiley, New York, 1967.

4. A. Bonami and J. L. Clerc, Sommes de Cesàro et multiplicateurs des développments en harmoniques sphériques, Trans. Amer. Math. Soc. 183 (1973), 223-263.

5. R. Coifman and G. Weiss, Analyse harmonique noncommutative sur certains èspaces homogènes, Lecture Notes in Math., vol. 242, Springer-Verlag, Berlin and New York, 1971.

6. W. C. Connett and A. L. Schwartz, A multiplier theorem for ultraspherical series, Studia Math. 51 (1974), 51-70.

7. $ـ$ A multiplier theorem for Jacobi expansions, Studia Math. 52 (1975), 243-261. (See also $A$ correction to the paper "A multiplier theorem for Jacobi expansions", Studia Math. 54 (1975), 197.)

8. The theory of ultraspherical multipliers, Mem. Amer. Math. Soc. No. 183, 1977.

9. G. Gasper, Positivity and the convolution structure for Jacobi series, Ann. of Math. 93 (1971), 112-118.

10. Banach algebras for Jacobi series and positivity of a kernel, Ann. of Math. 95 (1972), 261-280. 
11. G. Gasper and W. Trebels, A characterization of localized Bessel potential spaces and applications to Jacobi and Hankel multipliers (preprint).

12. __ Multiplier criteria of Marcinkiewicz type for Jacobi expansions (preprint).

13. G. H. Hardy and J. E. Littlewood, A maximal theorem with function-theoretic applications, Acta Math. 54 (1930), 81-116.

14. T. Koornwinder, Jacobi polynomials. II, An analytic proof of the product formula, SIAM J. Math. Anal. 5 (1974), 125-137.

15. H. M. Macdonald, Note on the evaluation of a certain integral containing Bessel's functions, Proc. London Math. Soc. (2) 7 (1909), 142-149.

16. U. Neri, Singular integrals, Lecture Notes in Math., vol. 200, Springer-Verlag, Berlin and New York, 1971.

17. H. Pollard, The mean convergence of orthogonal series. III, Duke Math. J. 16 (1949), 189-191.

18. E. Stein, Topics in harmonic analysis related to the Littlewood-Paley theory, Ann. of Math. Studies, no. 63, Princeton Univ. Press, Princeton, N. J., 1970.

19. E. Stein and G. Weiss, Introduction to Fourier analysis on Euclidean spaces, Princeton Univ. Press, Princeton, N. J., 1971.

20. G. Szegö, Orthogonal polynomials, third ed., Amer. Math. Soc. Colloq. Publ., vol. 23, Amer. Math. Soc., Providence, R. I., 1967.

21. G. N. Watson, $A$ treatise on the theory of Bessel functions, second ed., Cambridge Univ. Press, New York, 1966.

22. A. Zygmund, Trigonometric series, second ed., Cambridge Univ. Press, New York, 1968.

Department of Mathematics, University of Missouri, St. LoUts, Missouri 63121 